\title{
Treating many-body quantum systems by means of classical mechanics
}

\author{
Andrey R. Kolovsky
}

\begin{abstract}
Many-body physics of identical particles is commonly believed to be a sovereign territory of Quantum Mechanics. The aim of this contribution is to show that it is actually not the case and one gets useful insights into a quantum manybody system by using the theory of classical dynamical systems. In the contribution we focus on one paradigmatic model of many-body quantum physics - the BoseHubbard model which, in particular, describes interacting ultracold Bose atoms in an optical lattice. We show how one can find/deduce the energy spectrum of the Bose-Hubbard model by using a kind of the semiclassical approach.
\end{abstract}

\section{Introduction}

The semiclassical methods are known to be a powerful tool in studying quantum systems. They use information about classical dynamics of the system to predict its quantum dynamics or find the energy spectrum. Besides practical aspect, these methods also contribute to our understanding of subtle relation between the quantum and classical mechanics - an issue which might be even more important. Until now the overwhelming majority of semiclassical studies have been done for singleparticle problems. Yet, there is other type of problems which can be addressed by using the same kind of ideas - these are dynamical and spectral properties of an ensemble of identical particles. In this contribution we shall give an example of application of 'semiclassical methods' to one of the paradigm models of the manybody physics - the Bose-Hubbard (BH) model. This model describes, in particular, ultracold bosonic atoms optical lattices [6], with a unique for the many-body physics experimental control over the model parameters [7].

Andrey R. Kolovsky

L.V.Kirensky Institute of Physics of Siberian Branch of Russian Academy of Sciencies, 660036 Krasnoyarsk, Russia. e-mail: andrey.r.kolovsky@gmail.com 


\section{Bose-Hubbard model}

Denoting by $\hat{a}_{l}$ and $\hat{a}_{l}^{\dagger}$ the bosonic annihilation and creation operators, $\left[\hat{a}_{l}, \hat{a}_{l^{\prime}}^{\dagger}\right]=$ $\hbar \delta_{l, l^{\prime}}$, and by $\hat{n}_{l}=\hat{a}_{l}^{\dagger} \hat{a}_{l}$ the number operator, the Bose-Hubbard Hamiltonian reads

$$
\widehat{H}=\sum_{l=1}^{L} \varepsilon_{l} \hat{n}_{l}-\frac{J}{2} \sum_{l=1}^{L}\left(\hat{a}_{l+1}^{\dagger} \hat{a}_{l}+\text { h.c. }\right)+\frac{U}{2} \sum_{l=1}^{L} \hat{n}_{l}\left(\hat{n}_{l}-1\right) .
$$

In this Hamiltonian $\varepsilon_{l}$ are the on-site energies, $J$ is the hopping matrix element, and $U$ the microscopic interaction constant. Having in mind cold Bose atoms in the one-dimensional optical lattice, the constant $U$ is mainly determined by the $s$ wave scattering length for neutral atoms, and the constant $J$ by the lattice depth [6]. In laboratory experiments both the scattering length and the lattice depth can be varied in large intervals, which affords practically arbitrary ratio $U / J$. Notice, that the Hamiltonian (1) preserves the total number of particles (atoms), which we denote by $N$.

A remark concerning the boundary condition is in order. We assume a uniform system (i.e., no spatial dependence for the on-site energies), for which we shall use the periodic boundary condition if $L \geq 3$. In this case the Hamiltonian (1) can be rewritten in terms of the operators $\hat{b}_{k}$ and $\hat{b}_{k}^{\dagger}$,

$$
\hat{b}_{k}=\frac{1}{\sqrt{L}} \sum_{l} \exp \left(i \frac{2 \pi k}{L} l\right) \hat{a}_{l}, \quad \hat{b}_{k}^{\dagger}=\left(\hat{b}_{k}\right)^{\dagger} .
$$

Unlike the operators $\hat{a}_{l}\left(\hat{a}_{l}^{\dagger}\right)$, which annihilate (create) an atom in the Wannier states, operators (2) annihilate or create an atom in the Bloch states. Using the transformation (2) and dropping the first term in the Hamiltonian (1) (which is a constant for a uniform system) we have

$$
\widehat{H}=-J \sum_{k} \cos \left(\frac{2 \pi k}{L}\right) \hat{b}_{k}^{\dagger} \hat{b}_{k}+\frac{U}{2 L} \sum_{k_{1}, k_{2}, k_{3}, k_{4}} \hat{b}_{k_{1}}^{\dagger} \hat{b}_{k_{2}}^{\dagger} \hat{b}_{k_{3}} \hat{b}_{k_{4}} \tilde{\delta}\left(k_{1}+k_{2}-k_{3}-k_{4}\right)
$$

where $\tilde{\delta}$ is the periodic $\delta$-function, i.e., $\tilde{\delta}(k)$ equals unity if $k$ is a multiple of $L$ and zero otherwise. Depending on the addressed question this form of the $\mathrm{BH}$ model might be more convenient than Eq. (1). In particular, it follows from Eq. (3) that for $U=0$ the eigen-energies of the $\mathrm{BH}$ Hamiltonian are given by the equation,

$$
E_{j}=-J \sum_{k} \cos \left(\frac{2 \pi k}{L}\right) n_{k}, \quad \sum_{k} n_{k}=N .
$$

The total number of eigen-energies $E_{j}$ obviously coincides with dimension of the Hilbert space,

$$
\mathscr{N}=\frac{(N+L-1) !}{N !(L-1) !}
$$


which is obtained by counting all possible distributions of $N$ atoms among $L$ wells.

It is very difficult task to find energies $E_{j}$ if $U$ differs from zero. As it will be explained below, in general case we cannot find them analytically ${ }^{1}$ while numerically we are restricted to rather small system size because the dimension of the Hilbert space grows exponentially with $L$ and $N$. A way around these problems is to use 'semiclassical methods', where one is not bounded with small $N$ and $L$. To this end we introduce the classical counterpart of the quantum BH model.

\section{Classical Bose-Hubbard model}

Formally, the classical counterpart of the quantum BH model is obtained by rescaling the Hamiltonian (1) with respect to $N$ and identifying the creation and annihilation operators with $c$-number. This gives

$$
H=-\frac{J}{2} \sum_{l=1}^{L}\left(a_{l+1}^{*} a_{l}+\text { c.c. }\right)+\frac{g^{\prime}}{2} \sum_{l=1}^{L}\left|a_{l}\right|^{4},
$$

where the constant $g^{\prime}=U N$ is called the macroscopic interaction constant, to distinguish it from the microscopic interaction constant $U$. The Hamiltonian (6) generates classical trajectories according to the Hamilton equation of motion,

$$
i \frac{d}{d t} a_{l}=\frac{\partial H_{0}}{\partial a_{l}^{*}}=-\frac{J}{2}\left(a_{l+1}+a_{l-1}\right)+g^{\prime}\left|a_{l}\right|^{2} a_{l},
$$

which is known in the physical literature as the Discrete Nonlinear Schrödinger Equation (DNLSE). Let us remark that the conservation law for particle number takes the form of the norm conservation: $\sum_{l=1}^{L}\left|a_{l}\right|^{2}=1$.

Historically, Eq. (7) was deduced by using the mean-field approach, where the complex amplitudes $a_{l}$ have the meaning of order parameters. For this reason the classical Hamiltonian (6) is often referred to as the mean-field Hamiltonian. In the rest of this section we justify the Hamiltonian (6) rigorously, without appealing to the mean-field approximation. We shall follow an approach based on the notion of the Husimi function. ${ }^{2}$

Given $|\Psi(t)\rangle$ to be the many-body wave function of the quantum Hamiltonian, the Husimi function is defined as

$$
f(\mathbf{a}, t)=|\langle\mathbf{a} \mid \Psi(t)\rangle|^{2},
$$

where $|\mathbf{a}\rangle$ are the so-called coherent $S U(L)$ states[11],

\footnotetext{
${ }^{1}$ This should be opposed to the Fermi-Hubbard model, where the spectrum can be found analytically by using the Betha ansatz.

${ }^{2}$ A similar approach is based on the notion of the Wigner function [14, 13, 12]. The Husimi function, however, has an advantage that it is positively defined.
} 


$$
|\mathbf{a}\rangle=\frac{1}{\sqrt{N !}}\left(\sum_{l=1}^{L} a_{l} \hat{a}_{l}^{\dagger}\right)^{N}|v a c\rangle .
$$

Note that the Husimi function (8) is a function of $L$ complex amplitudes $a_{l}$ and the time $t$. In terms of the Husimi function (8) the Schrödinger equation for the wave function $|\Psi(t)\rangle$ takes the form

$$
\frac{\partial f}{\partial t}=\{H, f\}+O\left(\frac{1}{N}\right)
$$

where $\{\ldots, \ldots\}$ denotes the Poisson brackets, the $c$-number Hamiltonian $H$ is given in Eq. (6) and we refer the reader to the work [16] for the explicit form of terms which are inverse proportional to $N$. The crucial point in the presented derivation of the classical Hamiltonian is that Eq. (9) formally coincides with equation on the Husimi function of a single-particle system if one identifies $1 / N$ with the Planck constant. ${ }^{3}$ Thus one can use the common semiclassical theory to study the BH model. This theory relates the energy spectrum of the quantum $\mathrm{BH}$ model (1) to the phase-space structure of the classical $\mathrm{BH}$ model (6). We shall give examples in the subsequent sections.

To conclude this section we also display the classical counterpart of the Hamiltonian (3):

$$
H=-J \sum_{k=} \cos \left(\frac{2 \pi k}{3}\right) b_{k}^{*} b_{k}+\frac{g}{2} \sum_{k_{1}, k_{2}, k_{3}, k_{4}} b_{k_{1}}^{*} b_{k_{2}}^{*} b_{k_{3}} b_{k_{4}} \tilde{\delta}\left(k_{1}+k_{2}-k_{3}-k_{4}\right)
$$

where $g=U L / N$. Notice that the macroscopic interaction constant $g$ in Eq. (10) differs from the above introduced constant $g^{\prime}$ by the factor $L$. Often one uses the constant $g$ also in Eq. (7). In this case, however, the amplitude $a_{l}$ are normalized to $L$ but not to unity. To be certain, we shall characterize interactions by the constant $g=U N / L$ and restrict ourselves by the parameter region where $g \leq J$.

\section{Bose-Hubbard dimer}

We proceed with examples, where the simplest case corresponds to $L=2$ - the so-called $\mathrm{BH}$ dimer. Noticing that the $\mathrm{BH}$ system has two independent integrals of motion - the energy and the norm - we immediately conclude that the dimer is an integrable system. In fact, using the action-angle variables, $a_{l}=\sqrt{I_{l}} \exp \left(i \theta_{l}\right)$, and taking into account conservation of the norm, $I_{1}+I_{2}=1$, the original system of two degrees of freedom reduces to the following effective system of one degree of freedom,

\footnotetext{
${ }^{3}$ The effective Planck constant $\hbar_{\text {eff }}=1 / N$ should not be mismatched with the fundamental Planck constant $\hbar$ which we set to unity from now on. We also mention that within the discussed formalism $a_{l}$ and $a_{l}^{*}$ are the canonical variables, i.e., one does not interpret them as order parameters.
} 


$$
H_{e f f}=g I^{2}-J \sqrt{1-I^{2}} \cos (\theta), \quad|I| \leq 1,
$$

where $I=\left(I_{2}-I_{1}\right)$ and $\theta=\theta_{2}-\theta_{1} \cdot{ }^{4}$ For $g \neq 0$ the phase portrait of the system (11) resembles that of the mathematical pendulum. Small oscillations of this pendulum have the frequency

$$
\Omega=\sqrt{J^{2}+2 g J},
$$

which is know in the physical literature as the Josephson frequency. We mention, in passing, that Josephson's oscillations of cold atoms in a two-site optical potential were observed in the experiment [2].

Quantizing the effective system (11) in terms of $\hbar_{\text {eff }}=1 / N$ one obtains the energy spectrum of the $\mathrm{BH}$ dimer. As follows from the above analogy with the pendulum, the low-energy spectrum of the BH dimer should be equidistant with the level spacing given by the Josephson frequency $\Omega$, and the high-energy spectrum should consist of doubly degenerate levels, which correspond to the clockwise and counterclockwise rotations of the pendulum. These expectations are fully confirmed by the numerical analysis. Figure 1(a) shows the energy spectrum of the BH dimer as the function of the macroscopic interaction constant $g$ for $N=40$ where, to facilitate the comparison, we rescale the spectrum by using the Josephson frequency (12). The
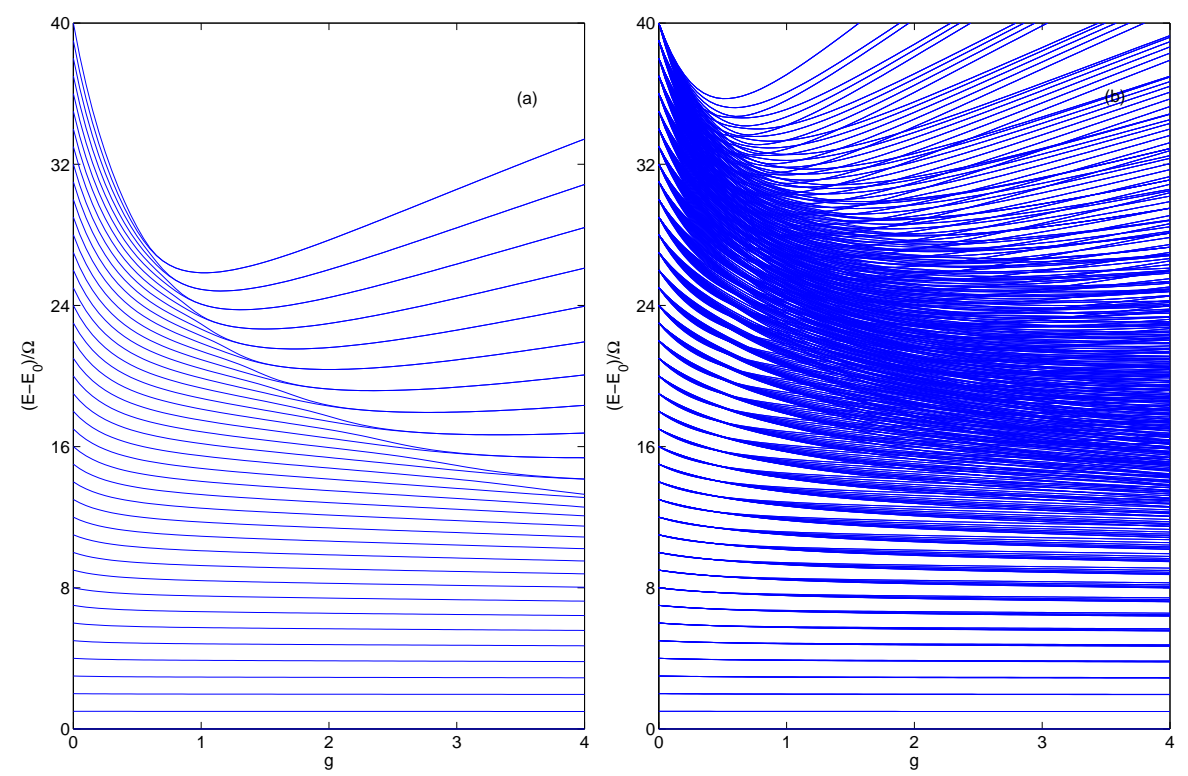

Fig. 1 Energy spectrum of the 2-site BH model, left, and the 3-site BH model, right, for $N=40$. The energy is measured relative to the ground energy $E_{0}$ and scaled with respect to the frequency $\Omega$ given in Eq. (12) and Eq. (15), respectively. The value of the hopping matrix element $J=1$.

\footnotetext{
${ }^{4}$ Using one more canonical transformation, $b_{1}=\left(a_{1}+a_{2}\right) / \sqrt{2}$ and $b_{2}=\left(a_{1}-a_{2}\right) / \sqrt{2}$, one gets a different form of the effective Hamiltonian, which is similar to Eq. (14) in Sec. 5. Naturally, this does not affect the final results.
} 
high- and low-energy regions, which are separated by the 'quantum separatrix', are clearly seen in Fig. 1(a). Let us also mention that in the limit $N \rightarrow \infty$ the density of state diverges at the separatrix [3].

\section{Bose-Hubbard trimer}

The case $L=3$ is more complicated because the classical BH trimer is a nonintegrable system with mixed phase space. It is largely an open question about the volumes of regular and chaotic components for a given energy shell $H(\mathbf{a})=E$. However, we can prove that the chaotic component vanishes at low energies and, hence, we are left with a stability island.

The proof involves several steps. First we rewrite the Hamiltonian (10) for $L=3$ in terms of the canonical variables $b_{k}$ and $b_{k}^{*}$. This gives

$$
H=-J \sum_{k=-1}^{1} \cos \left(\frac{2 \pi k}{3}\right) b_{k}^{*} b_{k}+\frac{g}{2} \sum_{k_{1}, k_{2}, k_{3}, k_{4}=1}^{-1} b_{k_{1}}^{*} b_{k_{2}}^{*} b_{k_{3}} b_{k_{4}} \tilde{\delta}\left(k_{1}+k_{2}-k_{3}-k_{4}\right) \text {. }
$$

Next we switch to the action-angle variables, $b_{k}=\sqrt{I_{k}} \exp \left(i \phi_{k}\right)$, and explicitly take into account that $\sum_{k} I_{k}=1$. This reduces our system of three degrees of freedom to the system of two degrees of freedom:

$$
\begin{array}{r}
H=(\delta+g)\left(I_{-1}+I_{+1}\right)+2 g I_{0} \sqrt{I_{-1} I_{+1}} \cos \left(\phi_{-1}+\phi_{+1}\right) \\
-g\left(I_{-1} I_{+1}+I_{-1}^{2}+I_{+1}^{2}\right)+2 g \sum_{ \pm} I_{\mp} \sqrt{I_{0} I_{ \pm 1}} \cos \left(2 \phi_{\mp 1}-\phi_{ \pm 1}\right),
\end{array}
$$

where $\delta=J[1-\cos (2 \pi / 3)], I_{0}=1-I_{-1}-I_{+1}$ and the phases $\phi_{ \pm 1}$ of variables $b_{ \pm 1}(t)$ are measured with respect to the phase of $b_{0}(t)$. The low-energy dynamics of the system (13), which is associated with the low-energy spectrum of the quantum system, implies $I_{ \pm 1} \ll I_{0}$. Keeping in the Hamiltonian (13) only the terms linear in $I_{ \pm 1}$, and using one more canonical transformation,

$$
\begin{gathered}
I=I_{+1}+I_{-1}, \quad \theta=\left(\phi_{+1}+\phi_{-1}\right) / 2, \\
M=\left(I_{+1}-I_{-1}\right) / 2, \quad \vartheta=\phi_{+1}-\phi_{-1},
\end{gathered}
$$

we end up with the effective Hamiltonian which locally describes the low-energy stability island:

$$
H_{e f f}=(\delta+g) I+g \sqrt{I^{2}-4 M^{2}} \cos (2 \theta), \quad|M| \leq I / 2 .
$$

Note that $H_{\text {eff }}$ does not include phase $\vartheta$ and, hence, the action $M$ is a constant of motion.

The obtained Hamiltonian (14) suffices to find the low-energy spectrum of the 3 -site BH model. To do this we integrate the system (14) by introducing new action, 
$\tilde{I}=(1 / 2 \pi) \oint I(\theta, E) d \theta$, and resolving the latter equation with respect to the energy. We get $E=\Omega \tilde{I}$ where

$$
\Omega=\sqrt{\delta^{2}+2 g \delta} .
$$

Finally, we quantize actions $\tilde{I}$ and $M$ in units of the effective Planck constant $\hbar_{\text {eff }}=1 / N$. This gives equidistant set of energy levels $E_{n}=E_{0}+\Omega n$, with $(n+1)$ degeneracy of the $n$th level. It should be stressed that the equidistant spectrum is an approximation which is valid until some critical energy $E_{c r}$. If we go to higher energy the spectrum becomes nonlinear and the degeneracy is removed, see Fig. 1(b). It is also seen in Fig. 1(b) that for high energies $E \approx E_{0}+\Omega N / 2$ the regular spectrum coexists with an irregular spectrum, which is consistent with the fact that highenergy energy shells of the classical 3-site system contain both regular and chaotic components.

\section{Many-site Bose-Hubbard model}

For $L \gg 1$ the role of chaos becomes even more important. Now majority of eigenstates of the BH model are chaotic states in the sense of Quantum Chaos [1, 15]. To clarify the meaning of 'majority of states' we discuss the density of states $\rho(E)$ of the quantum $\mathrm{BH}$ model for $L \gg 1$.

Let us for the moment $U=0$. Then the spectrum is known analytically, see Eq. (4). It follows from this equation that $\rho(E)$ has the region of support $|E| \leq J N$ and is peaked around $E=0$, see Fig. 2 (a). ${ }^{5}$ As $g$ is increased the whole distribution shifts to the right by the mean interaction energy $E_{\text {int }}=g N$ and becomes profoundly asymmetric, see panels (b) and (c) in Fig. 2. To relate the depicted distributions to the classical BH model we scale the energy $E$ and $\rho(E)$ with respect to $N$ and use the Weyl law. This gives

$$
\lim _{N \rightarrow \infty} N \frac{\rho(E / N)}{\mathscr{N}(N)}=\rho_{c l}(E)
$$

where $\mathscr{N}$ is given in Eq. (5), $\rho_{c l}(E)$ is the phase-space volume of the energy shell $H(\mathbf{a})=E$ of the classical BH model, and we implicitly assume that $\rho_{c l}(E)$ is normalized to unity (i.e., $\int \rho_{c l}(E) \mathrm{d} E=1$ ). For the considered $g=0,1,2$ the classical density of states $\rho_{c l}(E)$ is shown in the panels (d-f) in Fig. 2. A nice agreement with the quantum density of states indicates that $N=40$ is already large enough to drop the limit sign in Eq. (16). Let us also mention that for $L \gg 1$ and $g \ll J$ the function $\rho_{c l}(E)$ can be well approximated by the following simple equation,

$$
\rho_{c l}(E)=B \exp \left(A \sqrt{1-\frac{E^{2}}{J^{2}}}\right),
$$

\footnotetext{
${ }^{5}$ Slight asymmetry of $\rho(E)$ with respect to $E=0$ is related to the fact that $L$ is odd. For even $L$ (for example $L=6$ ) the distribution is perfectly symmetric, i.e., $\rho(E)$ is an even function of $E$.
} 
where $A=A(L)$ is the fitting parameter and $B$ the normalization coefficient. We shall use Eq. (17) together with Eq. (16) to approximate the density of states of the quantum BH model when performing statistical analysis of its energy spectrum.

The next step is to identify the borders of chaos in Fig. 2. To answer this question we again appeal to the classical $\mathrm{BH}$ model. Here the critical energy or, more exactly, crossover interval can be found by using Monte-Carlo simulations. In more details, we randomly generate initial condition $\mathbf{a}(t=0)$, evolve it in time by solving DNLSE, and determine whether the trajectory is regular or chaotic. It was found that for $L \geq 5$ and $g \sim J$ the crossover interval is close to the energy of the ground $E_{0} \equiv E_{\min } \approx-J+g / 2$, which corresponds to the extended initial condition $a_{l}(t=0) \approx 1 / \sqrt{L}$. For example, for $L=5$ and $g=1$ the crossover interval is $E_{\text {min }}+0.05 J<E<E_{\text {min }}+0.15 J$. It should be mentioned that there is another crossover interval which is close to the maximal energy $E_{\text {max }} \approx g L$, which corresponds to the localized initial condition $a_{l}(t=0) \approx \delta_{l, l^{\prime}}{ }^{6}$ In the context of cold atoms, however, only the lower critical energy is of interest because the right tail of the density of states of the single-band BH model usually overlaps with the spectrum originating from the second band of the many-bands $\mathrm{BH}$ model.
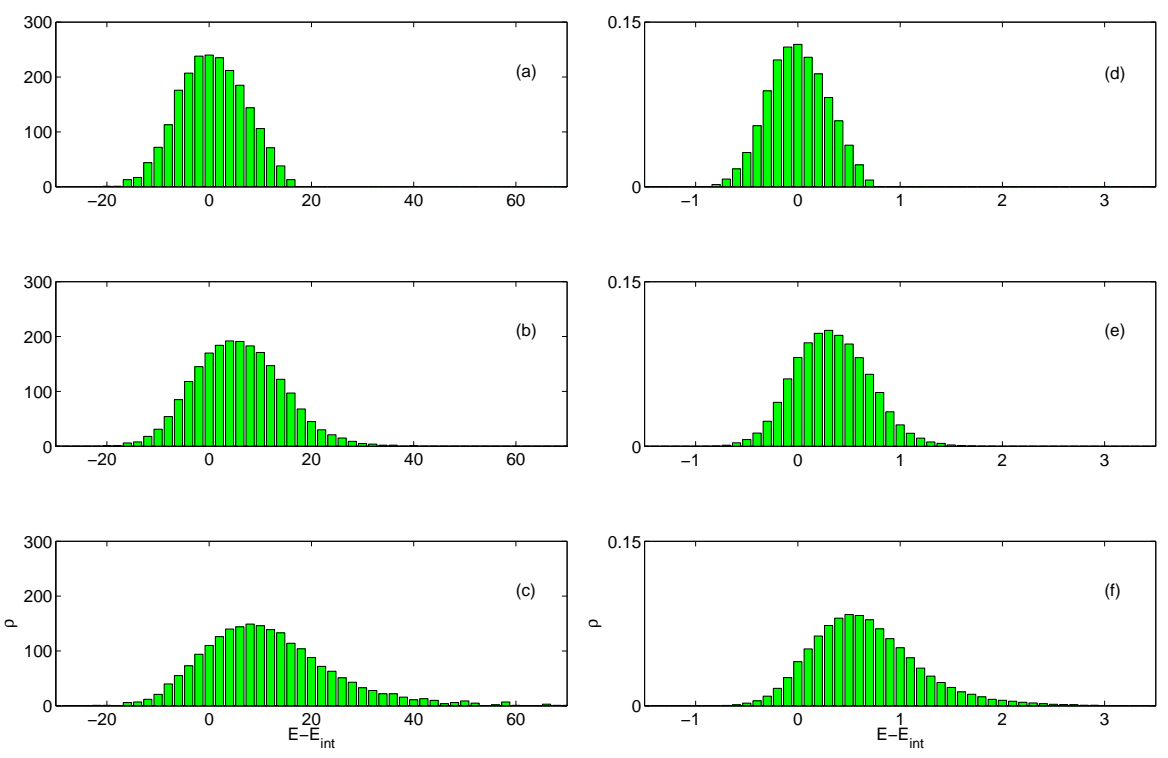

Fig. 2 Density of states of the 5-site BH model for $N=20$, panels (a-c), as compared to the classical 'density of states', panels (d-f). The energy is measured with respect to the mean interaction energy $E_{i n t}=g N$. The macroscopic interaction constant $g=0$, panels (a) and (d), $g=1$, panels (b) and (e), $g=2$, panels (c) and (f). The hopping matrix element $J=1$.

\footnotetext{
${ }^{6}$ Regular localized solutions of DNLSE are known as discrete solitons or breathers [5].
} 


\section{Statistical analysis of the energy spectrum}

As stated in Sec. 6, all states of the quantum BH model in the central part of the distribution $\rho(E)$ are chaotic states. This can be proved by statistical analysis of the eigenfunctions and eigen-energies, where the simplest test is the distribution $P(s)$ of the normalized distances $s$ between two nearest energy levels,

$$
s=\left(E_{j+1}-E_{j}\right) \rho\left(E_{j}\right) .
$$

If the states are chaotic, this distribution should obey the Wigner-Dyson statistics,

$$
P(s)=\frac{\pi}{2} s \exp \left(-\frac{\pi}{4} s^{2}\right) \text {. }
$$

The Wigner-Dyson statistics is usually opposed to the the Poisson statistics,

$$
P(s)=\exp (-s),
$$

which is typical for integrable systems. We note that in the numerical analysis it is more convenient to compare not distributions themselves but the integrated distributions

$$
I(s)=\int_{0}^{s} P(s) \mathrm{d} s^{\prime}
$$
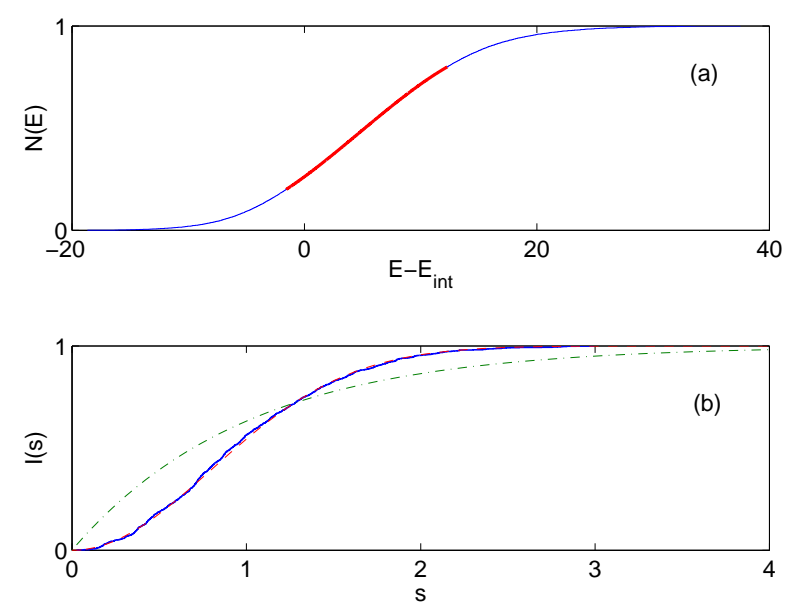

Fig. 3 Integrated density of states of the 5-site BH model for $g=1$ and $N=19$ (thin blue line in the upper panel), and the integrated level-spacing distribution $I(s)$ (solid blue line in the lower panel) as compared to the integrated Wigner-Dyson distribution (dashed red line) and the integrated Poisson distribution (dash-dotted green line). The hopping matrix element $J=1$. The energies are taken from the interval marked by the thick red line in the upper panel, that comprises 60 presents of the total number of states $\mathscr{N}=1771$. 
The solid line in the lower panel in Fig. 3 shows the integrated level-spacing distribution (21) for $L=5, N=19$, and $g=1$, where energies are taken from the energy interval marked by the thick line in the upper panel. ${ }^{7}$ An excellent agreement with the Wigner-Dyson distribution is noticed. Let us mention that this excellent agreement of the numerical data with Eq. (19) also indicates the relative volume of the chaotic component in the classical BH model to be close to unity in the considered energy interval. In this way the quantum and classical analysis of the $\mathrm{BH}$ model compliment each other. Furthermore, calculating $P(s)$ for different $g$ and approximating the result by the Berry-Robnik distribution, ${ }^{8}$ we can estimate the relative volumes of regular and chaotic components in the classical BH model. In particular, in the considered case $L=5$ the distribution $P(s)$ changes from almost perfect Poisson for $g=0.1$ to almost perfect Wigner-Dyson for $g=0.5$. Thus a transition to the developed chaos in the classical BH model (in the considered energy interval, of course) happens at $g=0.5$.

To conclude this section we briefly discuss the energy interval $E_{0}<E<E_{c r}$, where the energy spectrum is regular. We can find this regular spectrum by generalizing the approach of Sec. 5. In fact, the effective Hamiltonian (14) describes the coupling of the mode $k=0$ with the modes $k_{1}=1$ and $k_{2}=-1$. If $L>3$ the mode $k=0$ is also coupled to the modes $k_{1}=k$ and $k_{2}=-k$. Repeating the analysis of Sec. 5 we come to the effective Hamiltonian of the form (14) where the parameter $\delta=J[1-\cos (2 \pi / 3)]$ is substituted by the parameter $\delta_{k}=J[1-\cos (2 \pi k / L)]$. Thus the low energy spectrum of the system is given by a sum of equidistant spectra with the frequencies

$$
\Omega_{k}=\sqrt{2 g \delta_{k}+\delta_{k}^{2}}, \quad \delta_{k}=J[1-\cos (2 \pi k / L)] .
$$

If we restrict ourselves by small $k \ll L$, the frequencies (22) are approximated by

$$
\Omega_{\kappa}=\sqrt{2 g} \kappa, \quad \kappa=2 \pi k / L
$$

which is nothing else as the Bogoliubov dispersion relation for elementary excitations of the Bose-Einstein condensate.

\footnotetext{
${ }^{7}$ For the periodic boundary conditions (which are used throughout the paper) the quantum $\mathrm{BH}$ model possesses additional, pure quantum integral of motion - the total quasimomentum $\kappa=$ $2 \pi k / L$. Thus the whole spectrum can be decomposed into $L$ independent spectra labeled by $\kappa$. In Fig. 3 we choose $\kappa=2 \pi / L$ subspace. The results for other $\kappa$ look similar, except the case $\kappa=0$ where one should take into account the odd-even symmetry of the eigenstates.

${ }^{8}$ Berry-Robnik's statistics gives level-spacing distribution for a system with mixed phase space and interpolates between Poisson and Wigner-Dyson statistics.
} 


\section{Conclusion}

The presented results give the following picture of quantum-classical correspondence between the classical (6) and quantum (1) BH systems.

The low-energy trajectories of the classical system are regular and encircle the $2 L$-dimensional invariant torus. Topologically, this multi-dimensional torus is a tensor product of $L / 2$ four-dimensional tori defined by the effective Hamiltonians (14) with $\delta$ substituted by $\delta_{k}=J[1-\cos (2 \pi k / L)$. Quantizing these tori in terms of the effective Planck's constant $\hbar_{\text {eff }}=1 / N$ we obtain the lower-energy spectrum of the quantum system, which for $L \gg 1$ coincides with the Bogoliubov spectrum of elementary excitations above the ground state. Notice that, since we restrict ourselves to values of the macroscopic interaction constant $g$ of the same order as the hopping matrix element $J$ and assume $N \gg 1$, we have $U \ll J$. Thus the ground state of the system is a super-fluid state. It is an open question whether one can extend the semiclassical analysis into the region of large $g$, where the ground state of the system is a Mott insulator.

As we go to higher energies the invariant tori become gradually destroyed. This means that energy shells of the classical BH model contain both the regular and chaotic components and, depending on the initial condition, the classical trajectory is either regular or chaotic. With respect to the quantum BH model this is the most subtle case because the energy spectrum becomes a mixture of the regular spectrum, which is a reminiscent of the Bogoliubov spectrum, and an irregular spectrum.

With further increase of the energy the classical BH model shows a transition to the developed chaos and the spectrum of the quantum system becomes fully irregular. To avoid any misinterpretations we note that the term 'irregular' does imply the eigen-energies to be random numbers. On the contrary, there are important correlations between positions of the energy levels which are reflected, in particular, in the Wigner-Dyson distribution for distances between the nearest levels. Because the same correlations are present for eigenvalues of a random matrix, the meaning of the term 'irregular' is similarity of the spectrum with spectrum of random matrices.

In the work we focussed on the energy spectrum and did not pay much attention to the eigenfunctions. It was shown in Ref. [8] that eigenfunctions of the BH model also possess universal properties reflected, in particular, in the Breit-Wigner distribution for the local density of states. This has important consequences for transport phenomena with cold atoms. For example, if we address Bloch oscillations ${ }^{9}$ of interacting Bose atoms, we fnd that they irreversibly decay $[4,10]$. Remarkably, this quantum dynamics is perfectly reproduced by solving classical Eq. (9) on the truncated Husimi function [9]. This result provides one more example of successful treating of a many-body system by means of classical mechanics.

\footnotetext{
${ }^{9}$ Bloch oscillations are dynamical response of the system to an external static field. For noninteracting atoms these would be periodic oscillation of the mean atomic momentum with the Bloch frequency which is proportional to the field strength.
} 


\section{References}

1. Chaos and quantum physics. North-Holland, Amsterdam (1991)

2. Albiez, M., Gati, R., Fölling, J., Hunsmann, S., Cristiani, M., K.Oberthaler, M.: Direct observation of tunneling and nonlinear self-trapping in a single bosonic Josephson junction. Phys. Rev. Lett. 95, 010,402 (2005)

3. Bastidas, V.M., Engelhardt, G., Perez-Fernandez, P., Vogl, M., Brandes T.: Critical quasienergy states in driven many-body systems. Phys. Rev. A 90, 063628 (2014)

4. Buchleitner, A., Kolovsky, A.R.: Interaction-induced decoherence of atomic Bloch oscillations. Phys. Rev. Lett. 91, 253,002 (2003)

5. Campbell, D.K., Flach, S., Kivshar, Y.S.: Localizing energy through nonlinearity and discreteness. Phys. Today January 2004, 43 (2004)

6. Jaksch, D., Bruder, C., Cirac, J., Gardiner, C., Zoller, P.: Cold bosonic atoms in optical lattices. Phys. Rev. Lett. 81, 3108 (1998)

7. Jaksch, D., Zoller, P.: The cold atom Hubbard toolbox. Annals of Phys. 315, 52 (2005)

8. Kolovsky, A.R.: Conductivity with cold atoms in optical lattices. Journal of Statistical Mechanics: Theory and Experiment 2009, P02,018 (2009)

9. Kolovsky, A.R., Korsch, H.J., Graefe, E.M.: Bloch oscillations of Bose-einstein condensates: Quantum counterpart of dynamical instability. Phys. Rev. A 80, 023,617 (2009)

10. Meinert, F., Mark, M.J., Kirilov, E., Lauber, K., Weinmann, P., Gröbner, M., Nägerl, H.C.: Interaction-induced quantum phase revivals and evidence for the transition to the quantum chaotic regime in 1d atomic Dloch oscillations. Phys.Rev. Lett. 112, 193,003 (2014)

11. Perelomov, A.M.: Generalized coherent states and their applications. Springer, Berlin Heidelberg New York London Paris Tokyo (1986)

12. Polkovnikov, A.: Phase space representation of quantum dynamics. Annals of Phys. 325, 1790 (2010)

13. Sinatra, A., Lobo, C., Castin, Y.: The truncated Wigner method for Bose-condensed gases: limits of validity and applications. J. of Phys. B: At. Mol. Opt. Phys. 35, 3599 (2002)

14. Steel, M.J., Olsen, M.K., Plimak, L.I., Drummond, P.D., Tan, S.M., Collett, M.J., Walls, D.F., Graham, R.: Dynamical quantum noise in trapped Bose-einstein condensates. Phys. Rev. A 58, 4824 (1998)

15. Stöckmann, H.J.: Quantum Chaos. Cambridge University Press, Cambridge (1999)

16. Trimborn, F., Witthaut, D., Korsch, H.J.: Exact number conserving phase-space dynamics of the $M$-site Bose-Hubbard model. Phys. Rev. A 77, 043,631 (2008) 